\title{
Linked color imaging improves endoscopic diagnosis of active Helicobacter pylori infection
}

Authors

Institution
Osamu Dohi, Nobuaki Yagi, Yuriko Onozawa, Reiko Kimura-Tsuchiya, Atsushi Majima, Tomoko Kitaichi, Yusuke Horii, Kentaro Suzuki, Akira Tomie, Tetsuya Okayama, Naohisa Yoshida, Kazuhiro Kamada, Kazuhiro Katada, Kazuhiko Uchiyama, Takeshi Ishikawa, Tomohisa Takagi, Osamu Handa, Hideyuki Konishi, Yuji Naito, Yoshito Itoh

Department of Molecular Gastroenterology and Hepatology, Graduate School of Medical Science, Kyoto Prefectural University of Medicine, Kyoto, Japan submitted

5. February 2016

accepted after revision

17. May 2016

\section{Bibliography}

Dol http://dx.doi.org/

10.1055/s-0042-109049

Endoscopy International Open 2016; 04: E800-E805

(c) Georg Thieme Verlag KG Stuttgart · New York

E-ISSN 2196-9736

\section{Corresponding author}

\section{Osamu Dohi, MD, PhD}

Department of Molecular

Gastroenterology and

Hepatology

Graduate School of Medical

Science

Kyoto Prefectural

University of Medicine

465 Kawaramachi Hirokoji

Kamigyo-ku

Kyoto 602-8566

Japan

Phone: +81-75-251-5519

Fax: +81-75-251-0710

osamu-d@koto.kpu-m.ac.jp
Background and study aims: Linked color imaging (LCI) is a new image-enhanced endoscopy technique using a laser light source to enhance slight differences in mucosal color. The aim of this study was to compare the usefulness of $\mathrm{LCI}$ and conventional white light imaging (WLI) endoscopy for diagnosing Helicobacter pylori (H. pylori).

Patients and methods: We retrospectively analyzed images from 60 patients examined with WLI and LCI endoscopy between October 2013 and May 2014. Thirty patients had $H$. pylori infections, and other thirty patients tested negative for H. pylori after eradication therapy. Four endoscopists evaluated the 2 types of images to determine which was better at facilitating a diagnosis of $H$. pylori infection.

\section{Introduction}

$\checkmark$

Approximately half of the world's population is infected with Helicobacter pylori (H. pylori). Atrophic change and intestinal metaplasia often occur during long-term $H$. pylori infection, which can lead to gastric adenocarcinomas [1-3].

In Japan, national health insurance coverage for $H$. pylori eradication therapy to treat $\mathrm{H}$. pylori-associated gastritis finally became available in February 2013. However, in order for a patient to qualify for health insurance coverage, endoscopy must first be performed to diagnose $H$. pylori-associated gastritis [4].

Therefore, endoscopic examination is essential to make a diagnosis of $H$. pylori-associated gastritis. However, conventional white light imaging (WLI) endoscopy frequently correlates poorly with histopathological findings of $H$. pylori-induced gastritis [5-7]. Recently, a focus has been placed on analyzing endoscopic images of the diffuse redness of the fundic mucosa in $\mathrm{H}$. pylori-positive patients to diagnose infection. Using WLI, features of diffuse redness in the corpus and mucosal
Results: H. pylori infection was identified with LCI by enhancing the red appearance of the fundic gland mucosa. The accuracy, sensitivity, and specificity for diagnosing $H$. pylori infection using WLI were $74.2 \%, 81.7 \%$, and $66.7 \%$, respectively, while those for LCI were $85.8 \%, 93.3 \%$, and $78.3 \%$, respectively. Thus, the accuracy and sensitivity for LCI were significantly higher than those for WLI ( $P=0.002$ and $P=0.011$, respectively). The kappa values for the inter- and intraobserver variability among the 4 endoscopists were higher for LCI than for WLI.

Conclusions: $H$. pylori infection can be identified by enhancing endoscopic images of the diffuse redness of the fundic gland using $\mathrm{LCI}$. LCI is a novel image-enhanced endoscopy and is more useful for diagnosing $H$. pylori infection than is WLI.

swelling in the antrum were shown to be hallmarks of $H$. pylori infection [8]. Diffuse redness of the fundic mucosa was found significantly more frequently and covered more tissue area in $H$. pylori-positive patients (failed eradication) than in H. pylori-negative (successful eradication) patients in a multicenter prospective trial [9]. However, this study did not sufficiently evaluate the ability of non-expert endoscopists to diagnose $H$. pylori by evaluating these images.

We evaluated linked color imaging (LCI), a color enhancement feature available for LASEREO systems (FUJIFILM Co., Tokyo, Japan) that makes slight differences in mucosal color easy to recognize. We predicted that differences between mucosal color tones would become easy to recognize with LCI, thus supporting a diagnosis of diffuse redness of the fundic mucosa, which is difficult to detect using WLI. We then investigated the ability of both expert and non-expert endoscopists to diagnose $H$. pylori infections using LCI and WLI. 

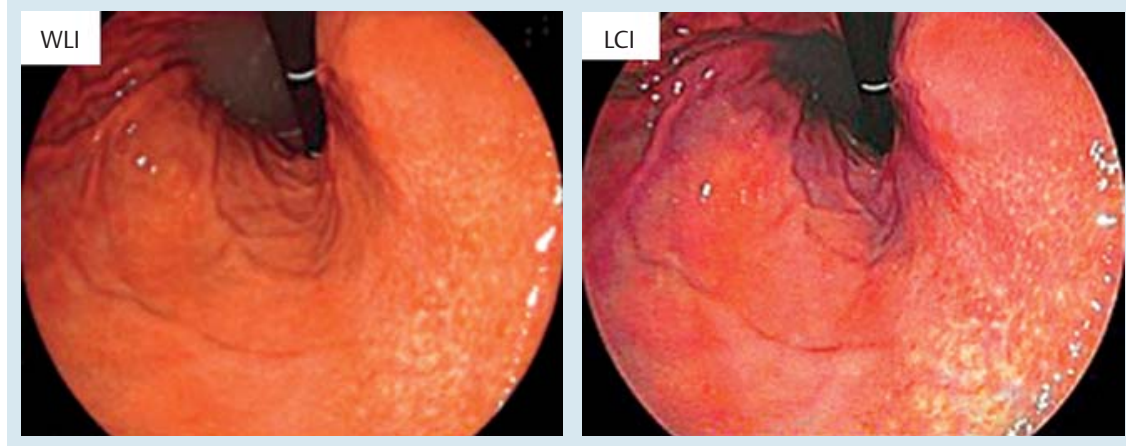

Fig. 1 Linked color imaging (LCI) principle. After reallocating the acquired color information on a mucosal surface or blood vessel by simultaneously irradiating narrow-band short wavelength light and white light, LCI both expands and reduces color information so that a reddish color becomes redder and a whitish color becomes whiter. R. red; G, green; B, blue. a White light imaging. $\mathbf{b} \mathrm{LCl}$.

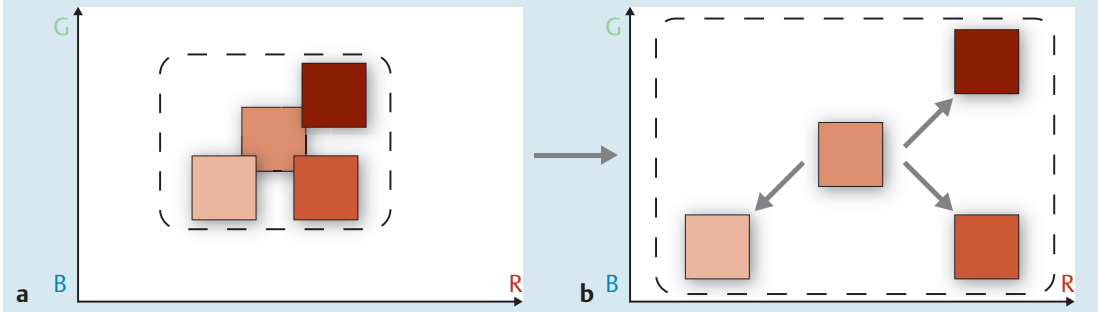

\section{Patients and methods}

\section{Patients}

This was a single-center retrospective study conducted in the Department of Molecular Gastroenterology and Hepatology, Kyoto Prefectural University of Medicine. The study was approved by the University Ethical Review Committee and performed out in accordance with the Helsinki Declaration of the World Medical Association. In addition, this study was registered in the University Hospital Medical Information Network Clinical Trials Registry (UMIN-CTR) as number UMIN0000021674. All patients provided written informed consents to undergo esophagogastroduodenoscopy (EGD) using both WLI and LCI at the University Hospital, Kyoto Prefectural University of Medicine.

Consecutive patients who underwent EGD and following examinations to determine their $H$. pylori infection status between October 2013 and October 2014 at the University Hospital, Kyoto Prefectural University of Medicine, were selected for this study. We evaluated both patients with $H$. pylori infection and those who tested negative for $H$. pylori infection after eradication therapy in order to evaluate and compare whether LCI and WLI could detect diffuse redness and diagnose $\mathrm{H}$. pylori infection accurately. The presence of $H$. pylori infection was determined by histologic examination, the rapid urease test PyloriTek $^{\circledR}$; Serim Research Corp., Elkhart, IN, USA), serum antibody test, or ${ }^{13} \mathrm{C}$-urea breath test (UBit ${ }^{\circledR}$; Otsuka, Tokyo, Japan). The absence of $H$. pylori infection was determined by the ${ }^{13} \mathrm{C}$-urea breath test and histological examination at least 2 months after $H$. pylori eradication therapy. The following patients were excluded from this study: (1) patients without $H$. pylori infection and without atrophic mucosa; (2) patients with histories of $H$. pylori eradication within the previous 1 year; (3) patients with histories of gastric surgery; and (4) patients with severe liver, renal, cardiopulmonary dysfunction, or blood disease including severe anemia.

\section{Endoscopic imaging}

All examinations were carried out with a high-definition EGL590WR endoscope corresponding to the LASEREO endoscopic system (FUJIFILM Co., Tokyo, Japan). In brief, this LASEREO system uses a semiconductor laser as the light source and has narrow-band light observation functions, BLI and LCI, without a customized optical filter. It uses 2 types of lasers, with 410-nm and $450-\mathrm{nm}$ wavelengths. Both bandwidths are $<2 \mathrm{~nm}$, in contrast to the bandwidth for NBI $(30 \mathrm{~nm})$ [10]. The laser with a 450-nm wavelength produces irradiated phosphor with an illumination similar to that produced by a xenon lamp. The combination of strong laser light with a wavelength of $450-\mathrm{nm}$ and fluorescent light provides illumination that is almost equivalent to that of WLI. LCI is a new image-enhanced technology that is intended to enhance slight color differences in the red region in the mucosa. This mode is based on the image captured under similar light conditions in BLI-bright; however, further post-image processing is later applied so that the slightly reddish color becomes much redder and slightly whitish color becomes much whiter than the colors appear under WLI ( $\bullet$ Fig. 1$)$. Ultimately, LCI improves the ability of the observer to recognize slight differences in mucosal color such as from inflammation or atrophy, compared to that with conventional WLI. WLI and LCI images were selected from the antegrade and retroflex views of the gastric body.

In the case of $H$. pylori infectious gastritis, WLI shows a slight reddish mucosa in the entire fundic gland ( $\bullet$ Fig. 2 a and $\bullet$ Fig. 2 b), while LCI shows a deep reddish (crimson) mucosa in the entire fundic gland ( $\bullet$ Fig. 3 a and $\bullet$ Fig. 3 b). In the cases of $H$. pylorinegative stomach after eradication therapy, WLI shows an orange/yellow mucosa in the entire fundic gland ( $\bullet$ Fig.4a and - Fig. 4 b), while LCI shows a light orange (white apricot) mucosa in the entire fundic gland ( $\bullet$ Fig. 5 a and $\bullet$ Fig. 5 b).

\section{Participating endoscopists}

Four endoscopists who were blinded to the clinical data evaluated all endoscopic images. None of them performed the EGDs in this study. Two expert endoscopists (expert A and B) had previously performed over 10,000 conventional EGDs, while the other 2 were non-expert endoscopists (non-expert C and D) who had previously performed less than 2000 conventional EGDs.

\section{Diagnostic evaluation}

Four endoscopists evaluated the images of the gastric fundic areas from the antegrade and retroflex views using each endoscopic modality (WLI and LCI). Images obtained with each modality were prepared for evaluation by placing them on a slide and 

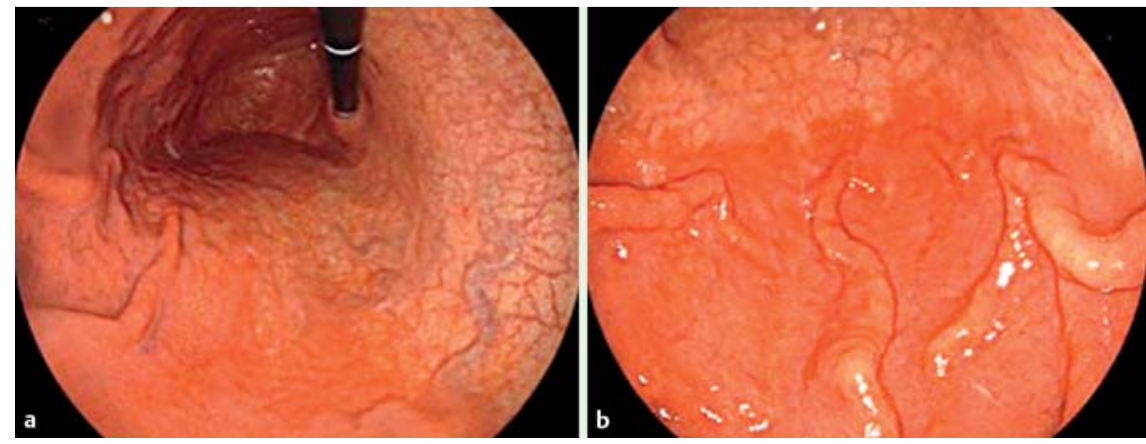

Fig. 2 Representative images of a case of $H$. pylori infectious gastritis. a White light imaging (WLI); gastric body from the retroflex view. $\mathbf{b}$ WLI; gastric body from the antegrade view.
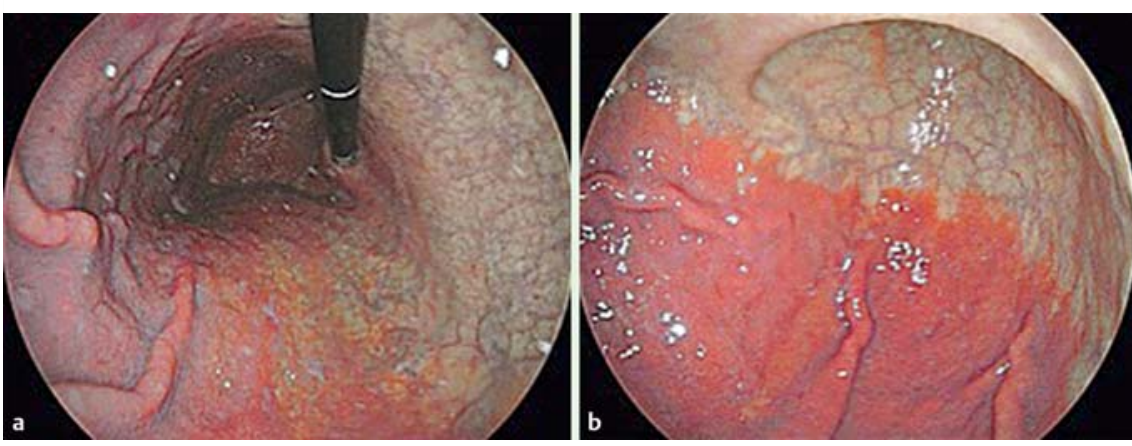

Fig. 3 Representative images of a case of $\mathrm{H}$. pylori infectious gastritis. a Linked color imaging (LCl); gastric body from the retroflex view. $\mathbf{b} \mathrm{LCl}$; gastric body from the antegrade view.

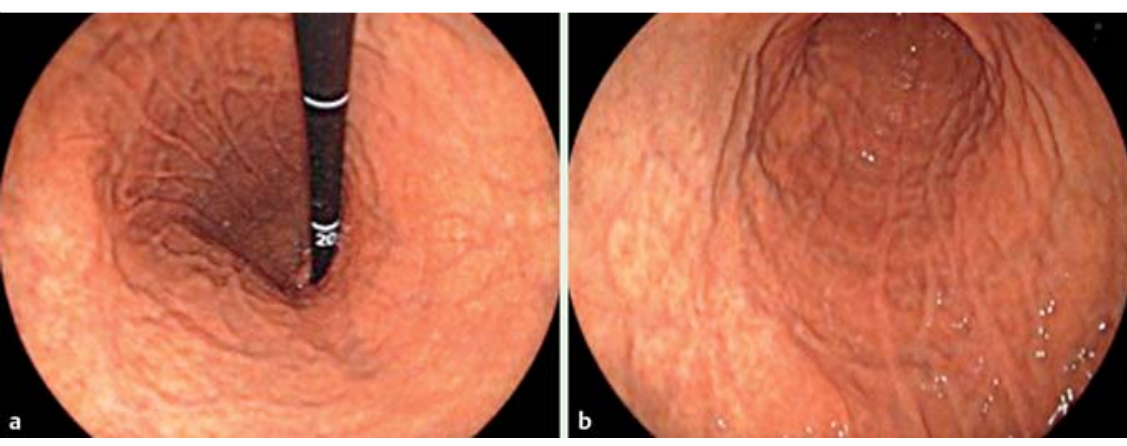

Fig. 4 Representative images of a case testing negative for $H$. pylori infection after eradication therapy. a White light imaging (WLI); gastric body from the retroflex view. $\mathbf{b}$ WLI; gastric body from the antegrade view.
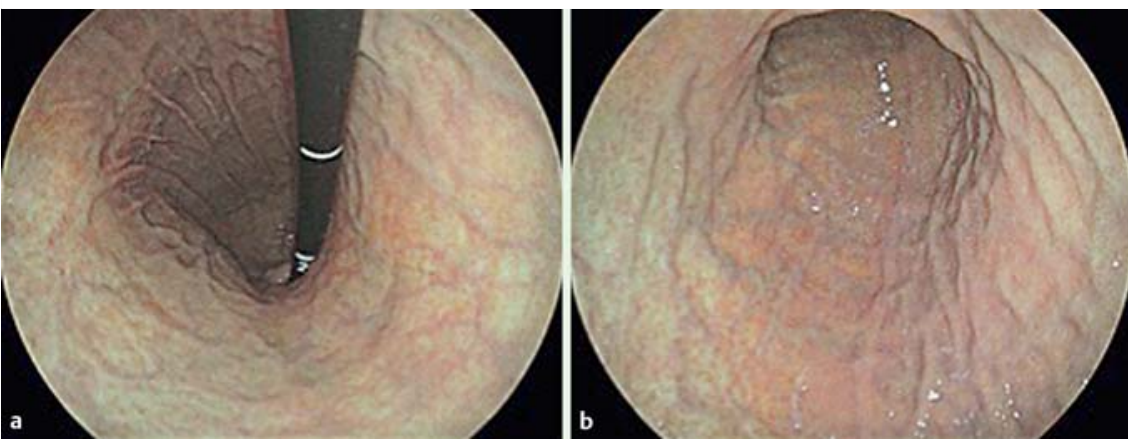

Fig. 5 Representative images of a case testing negative for $\mathrm{H}$. pylori infection after eradication therapy. a Linked color imaging (LCI); gastric body from the retroflex view. $\mathbf{b} \mathrm{LCl}$; gastric body from the antegrade view.

displaying them independently of the images obtained with the other endoscopic modality ( $\bullet$ Fig. 2 and $\bullet$ Fig. 3 ). The criterion for diagnosing $H$. pylori infection using both WLI and LCI was the presence of diffuse redness of the fundic mucosa. Using WLI, the diagnostic criterion of $H$. pylori infection is a marked or slight redness involving the entire mucosa of the fundic gland, while using $\mathrm{LCI}$, the diagnostic criterion is a deep red (crimson) color also involving the entire mucosa of the fundic gland.

In an independent experiment, we examined the interobserver and intraobserver variabilities of the endoscopists diagnosing $H$. pylori infection. All 4 endoscopists diagnosed the images from each case in the order of presentation in 1 day. One month later, the same images were presented in a different order for diagnosis by the same endoscopists.

\section{Calculation of sample size}

We evaluated the diagnostic differences in determining for $H$. pylori infection between WLI and LCI by the McNemar test in a pilot study of 24 patients. LCI was superior to WLI for diagnosing $H$. pylori infection in 8 patients (33\%), and the McNemar's odds ratio of LCI for WLI was 4.0. Thus, we needed 20 participants in order to detect the significant differences in diagnostic results with a probability (Power) of 0.8 and $\alpha$ error of 0.05 . Because the proportion of patients with a different diagnosis in the re- 
Table 1 Clinical features of patients.

\begin{tabular}{|c|c|c|c|}
\hline & $\begin{array}{l}\text { H. pylori-positive } \\
\mathrm{n}=\mathbf{3 0}\end{array}$ & $\begin{array}{l}\text { H. pylori-negative } \\
\mathrm{n}=30\end{array}$ & $P$ \\
\hline \multicolumn{4}{|l|}{ Gender } \\
\hline Male & 19 & 18 & \multirow{2}{*}{ n.s. } \\
\hline Female & 11 & 12 & \\
\hline Median age & 69.0 & 65.5 & n.s. \\
\hline \multicolumn{4}{|l|}{ Atrophic border ${ }^{1}$} \\
\hline$C-1, C-2, C-3$ & 5 & 9 & \multirow{3}{*}{ n.s. } \\
\hline $0-1,0-2$ & 22 & 17 & \\
\hline $0-3$ & 3 & 4 & \\
\hline
\end{tabular}

${ }^{1}$ Kimura-Takemoto classification system.

sponse of matched pairs was 33\%, the required sample size was set to 60 patients $(=20 / 0.33)$.

\section{Statistical analysis}

Continuous variables, such as patient age, were analyzed with the Mann-Whitney $U$ test. Comparisons of incidences between 2 groups such as sex and atrophic border, were analyzed with Pearson's chi-squared test or Fisher's exact test. McNemar's test was used to compare diagnostic accuracy, sensitivity, and specificity between WLI and LCI. The diagnostic accuracy of each method is presented as a percentage. The Wilson score method was used to measure the $95 \%$ confidence interval. Interobserver variability was quantified using the kappa statistic, which measures agreement over and above chance agreement. Kappa values of $<0.20$, $0.21-0.40,0.41-0.60,0.61-0.80$ and $>0.80$ are considered to indicate poor, fair, moderate, good and excellent agreement, respectively. $P<0.05$ was considered statistically significant. All statistical analyses were performed using SPSS software, Version 22.0 for Windows (IBM Japan, Ltd, Tokyo, Japan).

\section{Results}

\section{$\nabla$}

A total of 60 patients were evaluated in this study between October 2013 and October 2014. There were 30 patients with H. pylori infection and 30 patients who tested negative for $H$. pylori infection after eradication therapy. The clinical features of the patients are summarized in $\bullet$ Table 1.

The mean accuracy, sensitivity, specificity, positive predictive value (PPV), and negative predictive value (NPV) for LCI diagnoses for the 4 endoscopists were $85.8 \%, 93.3 \%, 78.3 \%, 81.2 \%$, and $92.2 \%$, respectively. On the other hand, the mean accuracy, sensitivity, specificity, PPV, and NPV for WLI diagnoses among the mean of 4 endoscopists were $74.2 \%, 81.7 \%, 66.7 \%, 70.6 \%$, and $75.5 \%$, respectively. The accuracy and sensitivity for LCI were significantly higher than those for WLI $(P=0.002$ and $P=0.011$, respectively). The performance data from the 4 endoscopists are shown in Table 2. The accuracy, sensitivity, specificity, NPV and PPV for LCI were higher for both experts and non-experts compared with WLI.

The kappa values of interobserver variability between the four endoscopists for WLI were fair to moderate. The kappa values of interobserver variability between the 4 endoscopists for LCI were as follows: expert to expert was excellent; the others were moderate to good ( Table 3 ). The kappa values of intraobserver variability for WLI were as follows: A and D were good, B was fair, and $C$ was moderate. The kappa values of intraobserver

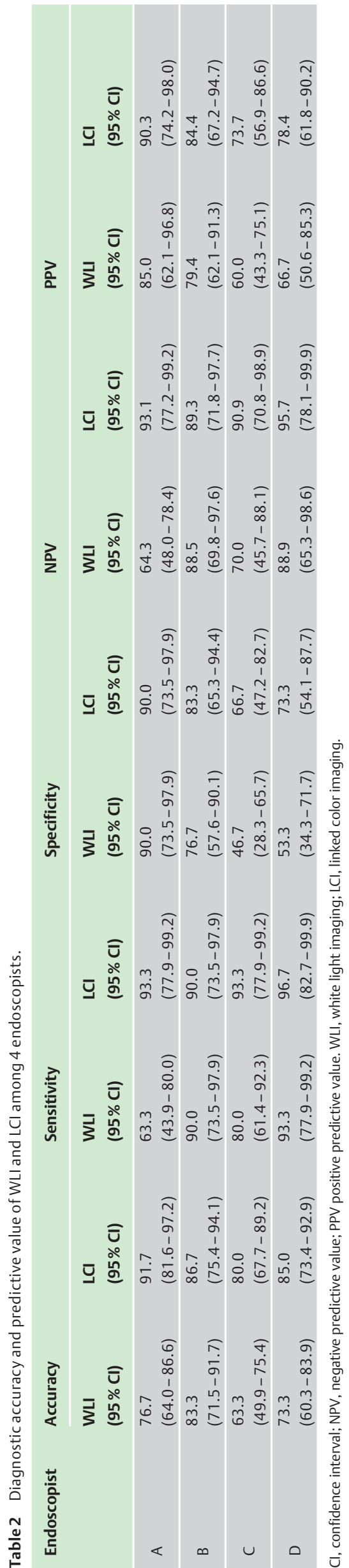


Table 3 Interobserver variability between expert and non-expert endoscopists.

\begin{tabular}{|c|c|c|c|c|c|c|}
\hline Endoscopist & A to $B$ & A to $C$ & $A$ to $D$ & B to $C$ & $B$ to $D$ & $C$ to $D$ \\
\hline Kappa values for WLI & 0.24 & 0.39 & 0.37 & 0.37 & 0.49 & 0.28 \\
\hline Kappa values for LCI & 0.85 & 0.45 & 0.75 & 0.45 & 0.75 & 0.45 \\
\hline
\end{tabular}

WLI, white light imaging; LCI, linked color imaging.

Table 4 Intraobserver variability among $4 r$ endoscopists.

\begin{tabular}{|lllll|}
\hline Endoscopist & A & B & C & D \\
\hline Kappa values for WLI & 0.73 & 0.39 & 0.47 & 0.64 \\
\hline Kappa values for LCI & 0.82 & 0.83 & 0.74 & 0.79 \\
\hline
\end{tabular}

WLI, white light imaging; LCl, linked color imaging.

variability for LCI were as follows: A and B were excellent, and the others were good ( Table4). The kappa values of interobserver and intraobserver variability for LCI were higher than those for WLI between all of the 4 endoscopists. In 7 cases with severe atrophic mucosa $(\mathrm{O}-3)$, there were $3 \mathrm{H}$. pylori-positive patients. All 3 patients were diagnosed as $H$. pylori-negative through the use of LCI by all 4 endoscopists.

\section{Discussion \\ $\nabla$}

To our knowledge, this is the first report to compare the usefulness of LCI for diagnosing $H$. pylori infection to that of WLI. Endoscopic diagnosis of $H$. pylori infection in gastric mucosa has been established. A regular arrangement of collecting venules is an endoscopic feature with high sensitivity and high specificity for detecting $H$. pylori-negative normal stomachs [11-13]. Studies using magnifying endoscopy or confocal laser endomicroscopy have shown endoscopic features associated with histopathologic findings related to $H$. pylori infection $[14,15]$. However, it was very difficult to observe and diagnose $H$. pylori infection using these methods, and assessment of endoscopic findings depended on the skill level of the endoscopists.

Endoscopic findings, such as diffuse or spotty redness, mucosal swelling, and swelling of the gastric area, can be used as diagnostic indices for $H$. pylori infection [8]. Diffuse redness in the fundic gland mucosa on histology was considered to reflect mucosal hyperemia due to inflammatory changes [16]. A strong correlation with an objective index of redness, the hemoglobin index (IHb), has been reported, suggesting that diffuse redness is the most important feature for diagnosing $H$. pylori infection [17]. Therefore, we hypothesized that LCI would detect diffuse redness more easily than would WLI.

The aim of our study was to evaluate whether LCI could detect this diffuse redness and diagnose $H$. pylori infection more accurately than could WLI. We excluded patients who did not have $H$. pylori infection, or atrophic mucosa, because the diagnostic accuracy of $H$. pylori infection by WLI is so high in these patients [8]. Therefore, we evaluated $H$. pylori infection status between $H$. pylori-positive patients and $H$. pylori-negative patients with atrophic mucosa after eradication.

The diagnostic accuracy of both experts and non-experts using LCI was superior to those for WLI. However, the kappa values for interobserver variability of LCI for comparisons between experts and non-experts or non-experts and non-experts was $<0.50$, which showed only moderate agreement. This was due to the use of recorded images and lack of experience of all endoscopists with LCI. Therefore, all endoscopists should practice LCI in real time. Biopsy-based methods for diagnosing $H$. pylori infection, such as the rapid urease test, histology, and bacterial culture, have a low sensitivity [18]. The accuracy of biopsy-based diagnosis using the Updated Sydney System [19] was dependent on the local mucosal conditions of the biopsy sites. If the biopsy specimens are taken at sites of atrophic mucosa and intestinal metaplasia, biopsy-based diagnosis may show false-negative results. LCI can evaluate the whole fundic gland area compared with biopsy-based methods. However, there were 3 false-negative cases of LCI diagnosis by all 4 endoscopists. In those cases, LCI showed no diffuse redness due to severe atrophic change of gastric corpus. Those are limitations of LCI diagnosis.

Our study has limitations. First, the number of patients was small and the data are from a single center. Second, this study is a limited evaluation of recorded images in selected patients. Third, the relationship between LCI and the Updated Sydney System was not assessed in this study. Therefore, WLI and LCI examination combined with the Updated Sydney System will be needed for further multi-institutional studies with a large number of cases. In conclusion, LCI can identify active $H$. pylori infection by emphasizing the diffuse redness of fundic gland mucosa. These results suggest that $\mathrm{LCI}$ improves endoscopic diagnostic accuracy of $H$. pylori infection compared with WLI.

Competing interests: Yuji Naito received collaboration research funding from Fujifilm Medical Co., Ltd (J132001115, J132001139). Nobuaki Yagi and Yoshito Itoh had an affiliation with donation-founded department partially from Fujifilm Medical Co., Ltd. Fujifilm Medical Co., Ltd. had no role in the study design, conduct of the study, data collection, data interpretation or preparation of the report.

\section{Acknowledgements \\ $\nabla$}

We thank all members of the Department of Molecular Gastroenterology and Hepatology, Kyoto Prefectural University of Medicine Graduate School of Medical Science for helping with this study. We also thank Kubo Masahiro and all other members who assisted with procedures related to the FUJIFILM LASEREO system. 


\section{References}

1 Marshall BJ, Armstrong JA, McGechie DB et al. Attempt to fulfill Koch's postulate for pyloric campylobacter. Med J Aust 1985; 142: 436 - 439

2 Perez-Perez GI, Rothenbacher D, Brenner H. Epidemiology of Helicobacter pylori infection. Helicobacter 2004; 9 (Suppl. 01): $1-6$

3 Chey WD, Wong BC. Practice Parameters Committee of the American College of Gastroenterology. American College of Gastroenterology guideline on the management of Helicobacter pylori infection. Am J Gastroenterol 2007; 102: 1808-1825

4 Asaka M, Mabe K. Strategies for eliminating death from gastric cancer in Japan. Proc Jpn Acad Ser B Phys Biol Sci 2014; 90: 251 - 258

5 Laine L, Cohen $H$, Sloane $R$ et al. Interobserver agreement and predictive value of endoscopic findings for $\mathrm{H}$. pylori and gastritis in normal volunteers. Gastrointest Endosc 1995; 42: 420 - 423

6 Khakoo SI, Lobo AJ, Shepherd NA et al. Histological assessment of the Sydney classification of endoscopic gastritis. Gut 1994; 35: $1172-$ 1175

7 Bah A, Saraga E, Armstrong D et al. Endoscopic features of Helicobacter pylori-related gastritis. Endoscopy 1995; 27: 593-596

8 Kato T, Yagi N, Kamada T et al. Diagnosis of Helicobacter pylori infection in gastric mucosa by endoscopic features: a multicenter prospective study. Dig Endosc 2013; 25: 508 -518

9 Kato M, Terao S, Adachi K et al. Changes in endoscopic findings of gastritis after cure of $\mathrm{H}$. pylori infection: multicenter prospective trial. Dig Endosc 2013; 25: $264-273$

10 Yoshida N, Hisabe T, Inada Yet al. The ability of a novel blue laser imaging system for the diagnosis of invasion depth of colorectal neoplasms. J Gastroenterol 2014; 49: 73-80
11 Yagi K, Nakamura A, Sekine A et al. Endoscopic features of the normal gastric mucosa without Helicobacter pylori infection. Gastroenterol Endosc 2000; 42: 1977-1987. (in Japanese with an English abstract)

12 Yagi $K$. Endoscopic features and magnified views of the corpus in the Helicobacter pylori-negative stomach. Dig. Endosc 2001; $13: 34-35$

13 Yagi K, Nakamura A, Sekine A. Characteristic endoscopic and magnified endoscopic findings in the normal stomach without Helicobacter pylori infection. J Gastroenterol Hepatol 2002; 17: 39-45

14 Nakagawa S, Kato M, Shimizu Y et al. Relationship between histopathologic gastritis and mucosal microvascularity: observations with magnifying endoscopy. Gastrointest Endosc 2003; 58: 71 - 75

$15 \mathrm{Ji} \mathrm{R}$, Li YQ Gu XM et al. Confocal laser endomicroscopy for diagnosis of Helicobacter pylori infection: a prospective study. J Gastroenterol Hepatol 2010; 25: 700-705

16 Hattori T. On cell proliferation and differentiation of fundic mucosa of the golden hamster. Fractographic study combined with microscopy and 3H-thymidine autoradiography. Cell Tissue Res 1974; 148: $213-$ 226

17 Uchiyama K, Ida K, Okuda J et al. Correlation of hemoglobin index (IHb) of gastric mucosa with Helicobacter pylori (H.pylori) infection and inflammation of gastric mucosa. Scand J Gastroenterol 2004; 11: 1054 1060

18 Gisbert JP, Abraira V. Accuracy of Helicobacter pylori diagnostic tests in patients with bleeding peptic ulcer: a systematic review and meta-analysis. Am J Gastroenterol 2006; 101: 848-863

19 Dixon MF, Genta RM, Yardley JH et al. Classification and grading of gastritis. The Updated Sydney System. Am J Surg Pathol 1996; 20: 1161 1181 